Proyecciones Journal of Mathematics Vol. 30, No 1, pp. 123-136, May 2011.

Universidad Católica del Norte

Antofagasta - Chile

\title{
A note on the Jordan decomposition
}

\author{
Mauro Patrão* \\ Universidade de Brasília-DF, Brazil \\ Laércio Santos \\ Universidade Federal de São Carlos, Brazil \\ and \\ Lucas Seco \\ Universidade de Brasília-DF, Brazil \\ Received : November 2010. Accepted : December 2010
}

\begin{abstract}
The multiplicative Jordan decomposition of a linear isomorphism of $\mathbf{R}^{n}$ into its elliptic, hyperbolic and unipotent components is well know. One can define an abstract Jordan decomposition of an element of a Lie group by taking the Jordan decomposition of its adjoint map. For real algebraic Lie groups, some results of Mostow implies that the usual multiplicative Jordan decomposition coincides with the abstract Jordan decomposition. Here, for a semisimple linear Lie group, we obtain this fact by elementary methods. We also obtain the corresponding results for semisimple linear Lie algebras. Complete and simple proofs of these facts are lacking in the literature, so that the main purpose of this article is to fill this gap.
\end{abstract}

\footnotetext{
${ }^{*}$ Supported by CNPq grant 310790/09-3
} 


\section{Introduction}

The multiplicative Jordan decomposition of a linear isomorphism of $\mathbf{R}^{n}$ into its elliptic, hyperbolic and unipotent components is well know (see Section IX.7, p.430 of [2]). For a Lie group $G$, one can define an abstract Jordan decomposition of $g \in G$ by taking the Jordan decomposition of the adjoint map $\operatorname{Ad}(g)$. For real algebraic Lie groups, some results of Mostow (see Section 3 of [6]) implies that the usual multiplicative Jordan decomposition coincides with the abstract Jordan decomposition. Here, for a semisimple linear Lie group, we obtain this fact by elementary methods. We also obtain the corresponding results for semisimple linear Lie algebras. Complete and simple proofs of these facts are lacking in the literature, so that the main purpose of this article is to fill this gap. As a byproduct we obtain that a semisimple linear Lie group is the connected component of the identity of an algebraic group and hence closed. Our interest in this subject arose in the article [1], where we related the Jordan decomposition with the dynamics on the flag manifold.

We now describe the structure of the present article. Let $V$ be a finite dimensional real vector space and $T$ a linear map of $V$. The most usual Jordan decomposition writes $T$ as a commuting sum of a semisimple and a nilpotent maps. They are called the semisimple and nilpotent additive Jordan components of $T$ and are given as polynomials in $T$ (see Theorem 13, p.267 of [3] or Proposition 4.2, p.17 of [4]). One can go further and write the semisimple component as a commuting sum of an elliptic and a hyperbolic components which also commutes with the nilpotent component. When $T$ is invertible, there is an analogous multiplicative Jordan decomposition which writes $T$ as a commuting product of an elliptic, a hyperbolic and a unipotent components (see Section IX.7, p.430 of [2]). In Section 2, our main results show that the elliptic and hyperbolic components of both additive and multiplicative Jordan decomposition of $T$ are given as polynomials in $T$ and the same happens for the unipotent component.

One can extend these decompositions to the context of semisimple Lie algebras and groups in the following manner (see $[2,7,8,9]$ ). Let $\mathbf{g}$ be a semisimple Lie algebra and $G$ be a Lie group with Lie algebra g. Let ad $: \mathbf{g} \rightarrow \mathbf{g l}(\mathbf{g})$ be the adjoint representation of $\mathbf{g}$ and let $\mathrm{Ad}: G \rightarrow \mathrm{Gl}(\mathbf{g})$ be the adjoint representation of $G$. For $X \in \mathbf{g}$ we say that $X=E+H+N$ is an abstract Jordan decomposition of $X$ if $E, H, N \in \mathbf{g}$ commute, $\operatorname{ad}(E)$ is additively elliptic, $\operatorname{ad}(H)$ is additively hyperbolic and $\operatorname{ad}(N)$ is nilpotent. For $g \in G$ we say that $g=e h u$ is an abstract Jordan decomposition of $g$ 
if $e, h, u \in G$ commute, $\operatorname{Ad}(e)$ is elliptic, $\operatorname{Ad}(h)$ is hyperbolic and $\operatorname{Ad}(u)$ is unipotent. In Section 3, by using the results of Section 2, we provide a simple proof that, for an element $X$ of a linear semisimple Lie algebra g (or $g$ of a linear semisimple connected Lie group $G$ ), its three Jordan components lie again in the algebra (in the group). This fact implies that, for this class of algebras and groups, the usual linear Jordan decomposition coincides with the abstract Jordan decomposition. For real algebraic Lie groups, this fact was previously obtained by Mostow (see Section 3 of [6]). Here we obtain this fact by elementary methods and, in particular, we obtain that a linear semisimple connected Lie group $G$ is the connected component of the identity of an algebraic group (see Proposition 3.5).

\section{General linear algebra and group}

We first introduce some preliminary definitions and notations. Defining the complex vector space $V_{\mathbf{C}}=\{u+i v: u, v \in V\}$ we have that $V \subset V_{\mathbf{C}}$. For $X \in \mathbf{g l}(V)$ we put $X(u+i v)=X u+i X v$ so that $\mathbf{g l}(V) \subset \operatorname{gl}\left(V_{\mathbf{C}}\right)$. Since the determinant of $g \in \mathrm{Gl}(V)$ seen as an operator of $V$ or $V_{\mathbf{C}}$ coincide we also have that $\operatorname{Gl}(V) \subset \operatorname{Gl}\left(V_{\mathbf{C}}\right)$. Let $X \in \operatorname{gl}(V)$. As usual, we say that $X$ is semisimple if it is diagonalizable in $V_{\mathbf{C}}$ and that $X$ is nilpotent if there exists $n \in N$ such that $X^{n}=0$. We say that $X$ is elliptic (hyperbolic) in the additive case if it is semisimple and its eigenvalues are purely imaginary (real). Now let $g \in \operatorname{Gl}(V)$. We say that $g$ is elliptic (hyperbolic) in the multiplicative case if it is semisimple and its eigenvalues have absolute value equal to one (are real positive). We say that $g$ is unipotent if $g-I$ is nilpotent. The proof of the following result is straightforward.

Lemma 2.1. Let $X, Y$ be two commuting linear maps of $V$.

1. If both $X, Y$ are semisimple, elliptic or hyperbolic, then $X+Y$ in the additive case (or $X Y$ in the multiplicative case) is semisimple, elliptic or hyperbolic.

2. If both $X, Y$ are nilpotent (or unipotent), then $X+Y$ is nilpotent (or $X Y$ is unipotent).

3. If $Y$ is simultaneously semisimple and nilpotent (or semisimple and unipotent) then $Y=0$ (or $Y=I$ ).

4. If $Y$ is elliptic and hyperbolic, then $Y=0$ in the additive case (or $Y=I$ in the multiplicative case). 
Denote by $\mathbf{F}[x]$ the ring of the polynomials in $x$ with coefficients in $\mathbf{F}=\mathbf{C}$ or $\mathbf{R}$. We denote by $\bar{p}(x)$ the polynomial whose coefficients are the conjugate of the coefficients of $p(x) \in \mathbf{F}[x]$. Thus $p(x) \in \mathbf{R}[x]$ if and only if $\bar{p}(x)=p(x)$. Let $X \in \mathbf{g l}(V)$ and consider the following ring homomorphism

$$
\mathbf{F}[x] \rightarrow \mathbf{g l}(V), \quad p(x) \mapsto p(X),
$$

where $p(x)=a_{0}+a_{1} x+\cdots+a_{m} x^{m}$ and $p(X)=a_{0} I+a_{1} X+\cdots+a_{m} X^{m}$. We denote by $\mathbf{F}(X)$ the image of this homomorphism. From now on, we will denote simply by $p$ both $p(x)$ and $p(X)$. It will be clear from the context which polynomial is considered. The kernel of the above homomorphism is the principal ideal generated by $p_{X}$, the so called minimal polynomial of $X$. Since $X \in \mathbf{g l}(V)$, it follows that $p_{X} \in \mathbf{R}[x]$. Since $p_{X} \in \mathbf{R}[x]$ we can factor it over $\mathbf{C}$ as

$$
p_{X}=p_{1} \bar{p}_{1} \cdots p_{l} \bar{p}_{l} p_{l+1} \cdots p_{n}
$$

where $p_{k}(x)=\left(x-\lambda_{k}\right)^{m_{k}}, \lambda_{k}$ has imaginary part for $k=1, \ldots, l$ and $\lambda_{k}$ is real for $k=l+1, \ldots, n$. Note that, since the characteristic polynomial of $X$ divides $p_{X}(x)$, we have that the eigenvalues of $X$ are $\lambda_{k}, \overline{\lambda_{k}}$, for $k=1, \ldots, n$.

Lemma 2.2. There exist polynomials $\pi_{k}$, where $1 \leq k \leq n$, and $l$ such that

(i) $\pi_{k} \in \mathbf{C}[x]$, for $1 \leq k \leq l$, and $\pi_{k} \in \mathbf{R}[x]$, for $l+1 \leq k \leq n$.

(ii) If $r \neq s$ then $\pi_{r} \pi_{s}, \bar{\pi}_{r} \pi_{s}$ and $\left(x-\lambda_{r}\right)^{m_{r}} \pi_{r}$ are multiples of $p_{X}$. We also have that $\bar{\pi}_{r} \pi_{r}$ is multiple of $p_{X}$ for $r=1, \ldots, l$.

(iii) $1=\sum_{k=1}^{l}\left(\pi_{k}+\bar{\pi}_{k}\right)+\sum_{k=l+1}^{n} \pi_{k}$.

Proof: For $1 \leq k \leq l$, we define the polynomials

$$
q_{k}=p_{1} \bar{p}_{1} \cdots p_{k} \widehat{\bar{p}}_{k} \cdots p_{l} \bar{p}_{l} p_{l+1} \cdots p_{n}
$$

whose conjugates are given by

$$
\bar{q}_{k}=p_{1} \bar{p}_{1} \cdots \widehat{p}_{k} \bar{p}_{k} \cdots p_{l} \bar{p}_{l} p_{l+1} \cdots p_{n},
$$

where the factor below - is omitted. For $l+1 \leq k \leq n$, we define the polynomials

$$
q_{k}=p_{1} \bar{p}_{1} \cdots p_{l} \bar{p}_{l} p_{l+1} \cdots \widehat{p}_{k} \cdots p_{n}
$$


Since the constant polynomials are the only polynomials dividing all $q_{k}$ and $\bar{q}_{k}$, where $1 \leq k \leq n$, it follows that the ideal generated by them is all of $\mathbf{C}[x]$. Thus there exists polynomials $a_{k}, b_{k}$ and $c_{k}$ such that

$$
1=\sum_{k=1}^{l}\left(a_{k} q_{k}+b_{k} \bar{q}_{k}\right)+\sum_{k=l+1}^{n} c_{k} q_{k}
$$

Adding the above equation with its conjugate and dividing by two, we can assume that $b_{k}=\bar{a}_{k}$ and $c_{k}=\bar{c}_{k}$. Defining the polynomials $\pi_{k}=a_{k} q_{k}$, $k=1, \ldots, l$, and $\pi_{k}=b_{k} q_{k}, k=l+1, \ldots, n$ we obtain the result.

Lemma 2.3. Applying the above polynomials to $X$ we have the following.

(i) $I=\sum_{k=1}^{l}\left(\pi_{k}+\bar{\pi}_{k}\right)+\sum_{k=l+1}^{n} \pi_{k}$.

(ii) If $r \neq s$, then $\pi_{r} \pi_{s}=0, \bar{\pi}_{r} \pi_{s}=0$ and $\left(X-\lambda_{r}\right)^{m_{r}} \pi_{r}=0$. We also have that $\bar{\pi}_{r} \pi_{r}=0$, for $r=1, \ldots, l$.

(iii) For $r=1, \ldots, n$ we have $\pi_{r}^{2}=\pi_{r}$.

Proof: Items (i) and (ii) are immediate from the previous lemma and the definition of the minimal polynomial. For item (iii), apply $\pi_{k}$ to both sides of item (i) and use item (ii).

Now we make the following remarks. By items (i) and (ii) of the above lemma we have that $V_{\mathbf{C}}$ is the direct sum of the images of the projections $\pi_{k}, \bar{\pi}_{k}$. Also, consider the linear maps

$$
T_{r}=\sum_{k=1}^{l}\left(a_{r k} \pi_{k}+b_{r k} \bar{\pi}_{k}\right)+\sum_{k=l+1}^{n} c_{r k} \pi_{k},
$$

where $r=1,2$ and $a_{r k}, b_{r k}, c_{r k}$ are polynomials in $X$. Again by the above lemma, we have that

$$
T_{1} T_{2}=\sum_{k=1}^{l}\left(a_{1 k} a_{2 k} \pi_{k}+b_{1 k} b_{2 k} \bar{\pi}_{k}\right)+\sum_{k=l+1}^{n} c_{1 k} c_{2 k} \pi_{k} .
$$

We now obtain the description of the additive Jordan components as polynomials. 
Theorem 2.4. Let $X \in \operatorname{gl}(V)$ and put $\lambda_{k}=u_{k}+i v_{k}$. Then $X$ can be written uniquely as a commutative sum $X=E+H+N$, where $E$ is elliptic, $H$ is hyperbolic and $N$ is nilpotent and they are given by the following real polynomials

(i) $E=\sum_{k=1}^{l}\left(i v_{k} \pi_{k}+{\overline{i v_{k}}}_{k}\right)$.

(ii) $H=\sum_{k=1}^{l} u_{k}\left(\pi_{k}+\bar{\pi}_{k}\right)+\sum_{k=l+1}^{n} u_{k} \pi_{k}$.

(iii) $N=\sum_{k=1}^{l}\left(\left(X-\lambda_{k}\right) \pi_{k}+\left(X-\bar{\lambda}_{k}\right) \bar{\pi}_{k}\right)+\sum_{k=l+1}^{n}\left(X-\lambda_{k}\right) \pi_{k}$.

Proof: Let $S=\sum_{k=1}^{l}\left(\lambda_{k} \pi_{k}+\bar{\lambda}_{k} \pi_{k}\right)+\sum_{k=l+1}^{n} \lambda_{k} \pi_{k}$. Note that $N=X-S$ and that $S=E+H$ and thus $X=E+H+N$. Using the remarks after Lemma 2.3, it is immediate that $S$ is semisimple, $E$ is elliptic, $H$ is hyperbolic and that

$$
N^{m}=\sum_{k=1}^{l}\left(\left(X-\lambda_{k}\right)^{m} \pi_{k}+\left(X-\bar{\lambda}_{k}\right)^{m} \bar{\pi}_{k}\right)+\sum_{k=l+1}^{n}\left(X-\lambda_{k}\right)^{m} \pi_{k} .
$$

Taking $m=\max _{k}\left\{m_{k}\right\}$, by item (ii) of Lemma 2.3, it follows that $N^{m}=0$.

For the uniqueness, consider the commuting sum $X=\widetilde{E}+\widetilde{H}+\widetilde{N}$, with $\widetilde{E}$ elliptic, $\widetilde{H}$ hyperbolic and $\widetilde{N}$ nilpotent. Define $\widetilde{S}=\widetilde{E}+\widetilde{H}$. Since $\widetilde{E}$ and $\widetilde{H}$ commute, by Lemma 2.1 , we have that $\widetilde{S}$ is semisimple. Since $E$, $H, N$ are polynomials in $X$, they commute with $\widetilde{E}, \widetilde{H}, \widetilde{N}$. Using that $X=S+N=\widetilde{S}+\widetilde{N}$, by Lemma 2.1 , we have that $S-\widetilde{S}=\widetilde{N}-N$ is both semisimple and nilpotent and thus $S=\widetilde{S}$ and $\widetilde{N}=N$. Now using that $S=E+H=\widetilde{E}+\widetilde{H}$, by Lemma 2.1 , we have that $E-\widetilde{E}=\widetilde{H}-H$ is both elliptic and hyperbolic and thus $E=\widetilde{E}$ and $\widetilde{H}=H$.

When $X$ is invertible, we denote it by $g \in \operatorname{Gl}(V)$ with eigenvalues $\lambda_{k}$. The following result provides the description of the multiplicative Jordan components as polynomials.

Theorem 2.5. Let $g \in \mathrm{Gl}(V)$ and put $\lambda_{k}=u_{k}+i v_{k}$. Then $g$ can be written uniquely as a commutative product $g=e h u$, where $e$ is elliptic, $h$ is hyperbolic and $u$ is unipotent and they are given by the following real polynomials

(i) $e=\sum_{k=1}^{l}\left|\lambda_{k}\right|^{-1}\left(\lambda_{k} \pi_{k}+\bar{\lambda}_{k} \pi_{k}\right)+\sum_{k=l+1}^{n}\left|\lambda_{k}\right|^{-1} \lambda_{k} \pi_{k}$.

(ii) $h=\sum_{k=1}^{l}\left|\lambda_{k}\right|\left(\pi_{k}+\bar{\pi}_{k}\right)+\sum_{k=l+1}^{n}\left|\lambda_{k}\right| \pi_{k}$. 
(iii) $u=I+N\left(\sum_{k=1}^{l}\left(\lambda_{k}^{-1} \pi_{k}+\overline{\lambda_{k}^{-1} \pi_{k}}\right)+\sum_{k=l+1}^{n} \lambda_{k}^{-1} \pi_{k}\right)$,

where $N$ is the nilpotent component of $g$. Furthermore, we have that $h=\mathrm{e}^{H}$, where

$$
H=\sum_{k=1}^{l} \log \left(\left|\lambda_{k}\right|\right)\left(\pi_{k}+\bar{\pi}_{k}\right)+\sum_{k=l+1}^{n} \log \left(\left|\lambda_{k}\right|\right) \pi_{k} .
$$

Proof: By the proof of Theorem 2.4 we have that $g=S+N$. Noting that $u=I+N S^{-1}$, we have that $S u=S+N=g$. It is immediate that $S=e h$, and thus $g=e h u$. Since $S, N$ commute and $N$ is nilpotent, it follows that $u$ is unipotent. Using the remarks after Lemma 2.3, it is immediate that $e$ is elliptic, that $h$ is hyperbolic and that $h=\mathrm{e}^{H}$.

For the uniqueness, consider the commuting product $g=\widetilde{e} \widetilde{h} \widetilde{u}$, with $\widetilde{e}$ elliptic, $\widetilde{h}$ hyperbolic and $\widetilde{u}$ unipotent. Define $\widetilde{S}=\widetilde{e} \widetilde{h}$. Since $\widetilde{e}$ and $\widetilde{h}$ commute, by Lemma 2.1 , we have that $\widetilde{S}$ is semisimple. Since $e, h, u$ are polynomials in $g$, they commute with $\widetilde{e}, \widetilde{h}, \widetilde{u}$. Using that $g=S u=\widetilde{S} \widetilde{u}$, by Lemma 2.1, we have that $\widetilde{S}^{-1} S=\widetilde{u} u^{-1}$ is both semisimple and unipotent and thus $S=\widetilde{S}$ and $\widetilde{u}=u$. Now using that $S=e h=\widetilde{e} \widetilde{h}$, by Lemma 2.1, we have that $\widetilde{e}^{-1} e=\widetilde{h} h^{-1}$ is both elliptic and hyperbolic and thus $e=\widetilde{e}$ and $\widetilde{h}=h$.

Example: In this example we display polynomials which give the additive and multiplicative Jordan components of the invertible linear map

$$
g=\left(\begin{array}{cccc}
1 & 1 & 0 & 0 \\
-1 & 1 & 0 & 0 \\
0 & 0 & 2 & 1 \\
0 & 0 & 0 & 2
\end{array}\right)
$$

which has minimal polynomial $p_{g}(x)=(x-(1+i))(x-(1-i))(x-2)^{2}$. By using the Euclidean algorithm, we obtain the following polynomials which satisfy Lemma 2.2

$$
\pi_{1}(x)=\frac{1}{4}(x-1+i)(x-2)^{2}, \quad \pi_{2}(x)=-\frac{1}{2}(x-3)\left(x^{2}-2 x+2\right) .
$$

Applying Theorem 2.4 we obtain, after factorization,

$$
E(x)=-\frac{1}{2}(x-2)^{2}, \quad H(x)=-\frac{1}{2} x^{3}+\frac{5}{2} x^{2}-4 x+4 .
$$


Applying Theorem 2.5 we obtain, after factorization,

$$
\begin{gathered}
e(x)=\frac{1}{4}(\sqrt{2}-2)\left(x^{3}+(\sqrt{2}-4) x^{2}+4(1-\sqrt{2}) x+2 \sqrt{2}-4\right), \\
h(x)=\frac{1}{2}(\sqrt{2}-2)\left(x^{3}-5 x^{2}+8 x-8-2 \sqrt{2}\right), \\
u(x)=\frac{1}{4} x\left(x^{2}-4 x+6\right) .
\end{gathered}
$$

One can use a mathematical software package to check that these polynomials give the correct additive and multiplicative Jordan components of $g$.

\section{Semisimple linear Lie algebras and groups}

In this section we will obtain the Jordan decomposition in semisimple linear Lie algebras and groups. When $\mathbf{g}=\mathbf{g l}(V)$ and $G=\mathrm{Gl}(V)$, we have that $\operatorname{ad}(X) Y=X Y-Y X$ and $\operatorname{Ad}(g) X=g X g^{-1}$.

\subsection{Additive Jordan decomposition}

Let $\mathbf{g} \subset \operatorname{gl}(V)$ be a semisimple Lie algebra of $\mathbf{g l}(V)$. Denote by $n(\mathbf{g})$ the normalizer of $\mathbf{g}$ in $\mathbf{g l}(V)$, that is,

$$
n(\mathbf{g})=\{X \in \mathbf{g l}(V): \operatorname{ad}(X) \mathbf{g} \subset \mathbf{g}\} .
$$

By definition $\mathbf{g}$ is an ideal in $n(\mathbf{g})$.

Consider the representation $\rho: \mathbf{g} \rightarrow \mathbf{g l}\left(V_{\mathbf{C}}\right)$, of $\mathbf{g}$ in $V_{\mathbf{C}}$, given by

$$
\rho(X)(v)=X v,
$$

for all $X \in \mathbf{g}$ and $v \in V_{\mathbf{C}}$. Since $\mathbf{g}$ is semisimple and $V_{\mathbf{C}}$ has finite dimension we have, by the Weyl decomposition theorem (Theorem 3.13.1, p.222 of [7]), that there exist subspaces $V_{1}, \ldots, V_{m}$ such that

$$
V_{\mathbf{C}}=V_{1} \oplus \cdots \oplus V_{m}
$$

and each $V_{k}, 1 \leq k \leq m$ is invariant and irreducible by $\rho$. For each $k=1, \ldots, m$, denote by $\mathbf{g}_{k}$ the subalgebra of $\mathbf{g l}(V)$ given by

$$
\mathbf{g}_{k}=\left\{X \in \operatorname{gl}(V): X\left(V_{k}\right) \subset V_{k} \text { and } \operatorname{tr}\left(\left.X\right|_{V_{k}}\right)=0\right\} .
$$


Since $\mathbf{g}$ is semisimple it follows that $\mathbf{g} \subset \mathbf{g}_{k}$. Let $\widetilde{\mathbf{g}}$ the following subalgebra of $\operatorname{gl}(V)$ :

$$
\widetilde{\mathbf{g}}=n(\mathbf{g}) \cap \mathbf{g}_{1} \cap \cdots \cap \mathbf{g}_{m} .
$$

We have that $\mathbf{g}$ is an ideal in $\widetilde{\mathbf{g}}$.

The following result is an adaptation for real semisimple Lie algebras of proof of Theorem 6.4, p.29 of [4].

Lemma 3.1. With the above notations, we have that $\mathbf{g}=\widetilde{\mathbf{g}}$.

Proof: Consider the representation $\widetilde{\rho}: \mathbf{g} \rightarrow \mathbf{g l}(\widetilde{\mathbf{g}})$ of $\mathbf{g}$ in $\widetilde{\mathbf{g}}$, given by $\widetilde{\rho}(X)(Y)=\operatorname{ad}(X) Y$, where $X \in \mathbf{g}$ and $Y \in \widetilde{\mathbf{g}}$. By the Weyl decomposition theorem (Theorem 3.13.1, p.222 of [7]), there exists a subspace $\mathbf{h} \subset \widetilde{\mathbf{g}}$ invariant by $\widetilde{\rho}$ such that

$$
\widetilde{\mathbf{g}}=\mathbf{g} \oplus \mathbf{h} .
$$

Since $\mathbf{h}$ is invariant by $\widetilde{\rho}$ and $\mathbf{g}$ is an ideal of $\widetilde{\mathbf{g}}$, it follows that $\operatorname{ad}(X) Y \in$ $\mathbf{g} \cap \mathbf{h}=\{0\}$, for all $X \in \mathbf{g}$ and $Y \in \mathbf{h}$.

Let $Y \in \mathbf{h}$. For each $1 \leq k \leq m$ and $v \in V_{k}$ we have that

$$
\rho(X) Y(v)=X Y(v)=Y X(v)=Y \rho(X) v
$$

for all $X \in \mathbf{g}$. By the Schur lemma, there exists $c \in \mathbf{C}$ such that $\left.Y\right|_{V_{k}}=c I_{k}$, where $I_{k}$ is the identity of $V_{k}$. We have that

$$
0=\operatorname{tr}\left(\left.Y\right|_{V_{k}}\right)=\operatorname{tr}\left(c I_{k}\right)=c \operatorname{dim} V_{k}
$$

so that $c=0$ and $\left.Y\right|_{V_{k}}=0$. Since $k$ is arbitrary, $Y=0$. It follows that $\mathbf{h}=\{0\}$, that is, $\widetilde{\mathbf{g}}=\mathbf{g}$.

Lemma 3.2. We have the following.

1. If $E \in \mathbf{g l}(V)$ is elliptic then $\operatorname{ad}(E)$ is elliptic.

2. If $H \in \mathbf{g l}(V)$ is hyperbolic then $\operatorname{ad}(H)$ is hyperbolic.

3. If $N \in \operatorname{gl}(V)$ is nilpotent, then $\operatorname{ad}(N)$ is nilpotent.

Proof: For items 1 and 2 , let $\left\{v_{1}, \ldots, v_{n}\right\}$ be a basis of $V_{\mathbf{C}}$ given by eigenvectors of a semisimple $S \in \mathbf{g l}(V)$. Let $\lambda_{1}, \ldots, \lambda_{n}$ be the respective eigenvalues. Consider the basis of $\mathbf{g l}\left(V_{\mathbf{C}}\right)$ given by $E_{r s}: V_{\mathbf{C}} \rightarrow V_{\mathbf{C}}$, $E_{r s}\left(v_{k}\right)=\delta_{j k} v_{r}$, where $\delta_{j k}$ is the Kronecker delta. We have that

$$
S E_{r s} v_{k}=S \delta_{j k} v_{r}=\lambda_{r} \delta_{j k} v_{r}=\lambda_{r} E_{r s} v_{k}
$$


and thus

$$
\operatorname{ad}(S) E_{r s} v_{k}=\left(\lambda_{r}-\lambda_{s}\right) E_{r s} v_{k}
$$

which shows that $E_{r s}$ is an eigenvector of $\operatorname{ad}(S)$ associated to the eigenvalue $\lambda_{r}-\lambda_{s}$. It is then immediate that $\operatorname{ad}(S)$ is elliptic (hyperbolic) when $S$ is elliptic (hyperbolic).

For the last item consider the linear map $L_{N}: \mathbf{g l}(V) \rightarrow \operatorname{gl}(V)$ given by $L_{N}(Y)=N Y, Y \in \operatorname{gl}(V)$. Since $L_{N}^{n}(Y)=N^{n} Y$, it follows that $L_{N}$ is nilpotent. Consider also the linear map $R_{N}: \operatorname{gl}(V) \rightarrow \operatorname{gl}(V)$ given by $R_{N}(Y)=Y N, Y \in \operatorname{gl}(V)$. Since $R_{N}^{n}(Y)=Y N^{n}$, it follows that $R_{N}$ is also nilpotent. Noting that $L_{N}$ and $R_{N}$ commute, and that $\operatorname{ad}(N)=L_{N}-R_{N}$, it follows that $\operatorname{ad}(N)$ is nilpotent.

We now obtain the main result of this subsection.

Theorem 3.3. Let $\mathbf{g}$ be a semisimple Lie subalgebra of $\mathbf{g l}(V)$ and $X \in \mathbf{g}$. The additive Jordan components of $X$ lie in $\mathbf{g}$.

Proof: Let $X=E+H+N$ be the additive Jordan decomposition of $X$. By Lemma 3.2 we have that $\operatorname{ad}(X)=\operatorname{ad}(E)+\operatorname{ad}(H)+\operatorname{ad}(N)$ is the additive Jordan decomposition of $\operatorname{ad}(X)$. By Theorem 2.4 it follows that $\mathbf{g}$ is invariant by the Jordan components of $\operatorname{ad}(X)$, since they are polynomials in $\operatorname{ad}(X)$. Thus, the Jordan components of $X$ lie in $n(\mathbf{g})$. Again by Theorem 2.4 it follows that $V_{k}$ is invariant by the Jordan components of $X$, since they are polynomials in $X$. Since $N$ is nilpotent, then $\left.N\right|_{V_{k}}$ is also nilpotent so that $\operatorname{tr}\left(\left.N\right|_{V_{k}}\right)=0$. Since $E$ is elliptic, then $\left.E\right|_{V_{k}}$ is also elliptic so that $\operatorname{tr}\left(\left.E\right|_{V_{k}}\right)$ is both real and pure imaginary and thus vanishes. It follows that $\operatorname{tr}\left(\left.H\right|_{V_{k}}\right)=0$, since $\operatorname{tr}\left(\left.X\right|_{V_{k}}\right)=0$. Hence the Jordan components of $X$ lie in $\mathbf{g}_{k}$, for each $k=1, \ldots, m$, showing that they lie in $\widetilde{\mathbf{g}}$. The result now follows from Lemma 3.1.

Using the previous result and Lemma 3.2 we have the next result, which proves also the existence of the abstract Jordan decomposition in $\mathbf{g}$.

Corollary 3.4. If $\mathbf{g}$ is a semisimple Lie subalgebra of $\mathbf{g l}(V)$ then the abstract and usual Jordan decompositions coincide.

\subsection{Multiplicative Jordan decomposition}

Let $\mathbf{g}$ be a semisimple Lie subalgebra of $\mathbf{g l}(V)$ and $G$ a connected Lie subgroup of $\mathrm{Gl}(V)$ with Lie algebra $\mathbf{g}$. We denote by $N(\mathbf{g})$ the normalizer 
of $\mathbf{g}$ in $\mathrm{Gl}(V)$ which is given by

$$
N(\mathbf{g})=\{g \in \operatorname{Gl}(V): \operatorname{Ad}(g) \mathbf{g}=\mathbf{g}\} .
$$

We have that $G$ is a normal subgroup of $N(\mathbf{g})$ since, by the connectedness of $G, N(\mathbf{g})$ is the normalizer of $G$ in $\mathrm{Gl}(V)$.

Consider the representation $\rho: \mathbf{g} \rightarrow \mathbf{g l}\left(V_{\mathbf{C}}\right)$ given in $(3.1)$ and the decomposition $V_{\mathbf{C}}=V_{1} \oplus \cdots \oplus V_{m}$ given in (3.2), such that each $V_{k}$ is invariant and irreducible by $\rho, 1 \leq k \leq m$. For each $k=1, \ldots, m$, denote by $G_{k}$ the subgroup of $\mathrm{Gl}(V)$ given by

$$
G_{k}=\left\{g \in \mathrm{Gl}(V): g\left(V_{k}\right) \subset V_{k} \mathrm{e} \operatorname{det}\left(\left.g\right|_{V_{k}}\right)=1\right\} \text {. }
$$

Since $G$ is connected and semisimple it follows that $G \subset G_{k}$. Consider the subgroup of $\mathrm{Gl}(V)$ given by

$$
\widetilde{G}=N(\mathbf{g}) \cap G_{1} \cap \cdots \cap G_{m}
$$

Proposition 3.5. Let $G$ be a connected semisimple Lie subgroup of $\mathrm{Gl}(V)$.

With the above notations, $\widetilde{G}$ is algebraic and its connected component of the identity is $G$. In particular, $G$ is closed.

Proof: We first show that $\widetilde{G}$ is algebraic. We start by showing that $N(\mathbf{g})$ is algebraic. Let $\mathbf{g}$ be given, as a subspace, by the kernel of a $T \in \operatorname{gl}(\operatorname{gl}(V))$. Consider $g \in \operatorname{Gl}(V)$, then it is well known $\widehat{g}=g^{-1} \operatorname{det}(g)$ is a polynomial in $g$. Then condition that $\operatorname{Ad}(g) \mathbf{g}=\mathbf{g}$ is clearly seen to be equivalent to $T(g X \widehat{g})=0$, for all $X \in \mathbf{g}$. Thus, taking a basis $\left\{X_{1}, \ldots, X_{n}\right\}$ of $\mathbf{g}$, the condition $\operatorname{Ad}(g) \mathbf{g}=\mathbf{g}$ is equivalent to the algebraic condition $T\left(g X_{r} \widehat{g}\right)=0$, for $r=1, \ldots, m$. To show that each $G_{k}$ is algebraic we choose a basis of $\left\{v_{1}, \ldots, v_{n}\right\}$ of $V_{\mathbf{C}}$ such that $\left\{v_{1}, \ldots, v_{l}\right\}$ is a basis of $V_{k}, l \leq n$. Denote by $z_{r s}(g)$ the $(r, s)$-entry of the matrix of $g \in \mathrm{Gl}(V)$ in this basis. It follows that $g V_{k} \subset V_{k}$ if and only if $z_{r s}(g)=0$ for $r>l, s \leq l$, and in this case we have

$$
\operatorname{det}\left(\left.g\right|_{V_{k}}\right)=\operatorname{det}\left(\left(z_{r s}(g)\right)_{1 \leq r, s \leq l}\right) .
$$

Now, since $G$ is connected, it is enough to show that its Lie algebra coincides with the Lie algebra of $\widetilde{G}$. Since the Lie algebra of $N(\mathbf{g})$ is $n(\mathbf{g})$ and the Lie algebra of $G_{k}$ is the subalgebra $\mathbf{g}_{k}$ given in (3.3), we have that the Lie algebra of $\widetilde{G}$ is given by (3.4). The result now follows by Lema 3.1. 
Lemma 3.6. We have the following.

1. If $e \in \operatorname{Gl}(V)$ is elliptic then $\operatorname{Ad}(e)$ is elliptic.

2. If $h \in \mathrm{Gl}(V)$ is hyperbolic then $\operatorname{Ad}(h)$ is hyperbolic.

3. If $u \in \mathrm{Gl}(V)$ is unipotent, then $\operatorname{Ad}(u)$ is unipotent.

Proof: For items 1 and 2, let $\left\{v_{1}, \ldots, v_{n}\right\}$ be a basis of $V_{\mathbf{C}}$ given by eigenvectors of a semisimple $s \in \mathrm{Gl}(V)$. Let $\lambda_{1}, \ldots, \lambda_{n}$ be the respective eigenvalues. Consider the basis of $\mathbf{g l}\left(V_{\mathbf{C}}\right)$ given by $E_{r s}: V_{\mathbf{C}} \rightarrow V_{\mathbf{C}}$, $E_{r s}\left(v_{k}\right)=\delta_{j k} v_{r}$, where $\delta_{j k}$ is the Kronecker delta. We have that

$$
\begin{aligned}
\operatorname{Ad}(s) E_{r s} v_{k} & =s E_{r s} s^{-1} v_{k}=s E_{r s} \lambda_{k}^{-1} v_{k}=\lambda_{k}^{-1} \delta_{j k} s v_{r} \\
& =\lambda_{r} \lambda_{s}^{-1} \delta_{j k} v_{r}=\lambda_{r} \lambda_{s}^{-1} E_{r s} v_{k} .
\end{aligned}
$$

which shows that $E_{r s}$ is an eigenvector of $\operatorname{Ad}(s)$ associated to the eigenvalue $\lambda_{r} \lambda_{s}^{-1}$. It is then immediate that $\operatorname{Ad}(s)$ is elliptic (hyperbolic) when $s$ is elliptic (hyperbolic).

For the last item, by Lemma IX.7.3 p.431 of [2], we have that $u=\mathrm{e}^{N}$ where $N \in \mathbf{g}$ is nilpotent. Since $\operatorname{Ad}(u)=\mathrm{e}^{\operatorname{ad}(N)}$ the result follows from the last item of Lemma 3.2.

We now obtain the principal result of this subsection.

Theorem 3.7. Let $G$ be a connected semisimple Lie subgroup of $\mathrm{Gl}(V)$ and $g \in G$. Then the multiplicative Jordan components of $g$ lie in $G$.

Proof: Let $g=e h u$ be the multiplicative Jordan decomposition of $g$. By Lemma 3.6 we have that $\operatorname{Ad}(g)=\operatorname{Ad}(e) \operatorname{Ad}(h) \operatorname{Ad}(u)$ is the multiplicative Jordan decomposition of $\operatorname{Ad}(g)$. By Theorem 2.5 it follows that $\mathrm{g}$ is invariant by the Jordan components of $\operatorname{Ad}(g)$, since they are polynomials in $\operatorname{Ad}(g)$. Thus, the Jordan components of $g$ lie in $N(\mathbf{g})$. Again by Theorem 2.5 it follows that $V_{k}$ is invariant by the Jordan components of $g$, since they are polynomials in $g$. Since $u$ is unipotent, then $\left.u\right|_{V_{k}}$ is also unipotent so that $\operatorname{det}\left(\left.u\right|_{V_{k}}\right)=1$. Since $e$ is elliptic and $h$ is hyperbolic, then $\left.e\right|_{V_{k}}$ is also elliptic and $\left.h\right|_{V_{k}}$ is also hyperbolic so that $\operatorname{det}\left(\left.e\right|_{V_{k}}\right)$ is real and have absolute value equal to one and $\operatorname{det}\left(\left.h\right|_{V_{k}}\right)$ is positive real. It follows that $\operatorname{det}\left(\left.e\right|_{V_{k}}\right)=\operatorname{det}\left(\left.h\right|_{V_{k}}\right)=1$, since $\operatorname{det}\left(\left.g\right|_{V_{k}}\right)=1$. Hence the Jordan components of $g$ lie in $G_{k}$, for each $k=1, \ldots, m$, showing that they lie in $\widetilde{G}$. By 
Proposition 3.5, it remains to show that the Jordan components of $g$ lie in the connected component of the identity of $\widetilde{G}$.

For the hyperbolic component, by Theorem 2.5, we have that $h=\mathrm{e}^{H}$, where $H \in \operatorname{gl}(V)$ is hyperbolic. Let $\left\{v_{1}, \ldots, v_{l}\right\}$ be a basis of $V$ such that $H v_{r}=\lambda_{r} v_{r}$. We have that $h^{n} v_{r}=\mathrm{e}^{n \lambda_{r}} v_{r}$, for all $n \in \mathbf{Z}$. In this basis, let $\left\{Q_{s}\right\}$ be the set of polynomials defining $\widetilde{G}$. Let $P_{s}$ be the polynomial obtained by restricting $Q_{s}$ to the diagonal matrices in this basis. Since $h^{n} \in \widetilde{G}$, we have that $P_{s}\left(\mathrm{e}^{n \lambda_{1}}, \ldots, \mathrm{e}^{n \lambda_{l}}\right)=0$, for all $n \in \mathbf{Z}$. By Lemma 1.142 p.116 of [5], we have that $P_{s}\left(\mathrm{e}^{t \lambda_{1}}, \ldots, \mathrm{e}^{t \lambda_{l}}\right)=0$, for all $t \in \mathbf{R}$. This shows that $e^{t H} \in \widetilde{G}$, for all $t \in \mathbf{R}$, so that $h$ lies in the connected component of identity of $\widetilde{G}$. For the unipotent component, by Lemma IX.7.3 p.431 of [2], we have that $u=\mathrm{e}^{N}$, where $N \in \operatorname{gl}(V)$ is nilpotent. Choosing a basis of $V$, we have that the $(r, s)$-entry of $u^{n}$ is a polynomial $p_{r s}(n)$ in $n \in \mathbf{Z}$, since $u^{n}=e^{n N}$ and $N$ is nilpotent. We have that $q_{j}(n)=Q_{j}\left(\left(p_{r s}(n)\right)_{1 \leq r, s \leq l}\right)$ is also a polynomial in $n$. Since $u^{n} \in \widetilde{G}$, we have that $q_{j}(n)=0$, for all $n \in \mathbf{Z}$, which implies that $q_{j}(t)=0$, for all $t \in \mathbf{R}$. This shows that $e^{t N} \in \widetilde{G}$, for all $t \in \mathbf{R}$, so that $u$ lies in the connected component of identity of $\widetilde{G}$.

Since $g$ is already in $G$ and $g=e h u$ it follows that $e$ lies in $G$, which completes the proof.

Using the previous result and Lemma 3.6 we have the next result, which proves also the existence of the abstract Jordan decomposition in $G$.

Corollary 3.8. If $G$ is a connected semisimple Lie subgroup of $\mathrm{Gl}(V)$ then the abstract and usual Jordan decompositions coincide.

\section{References}

[1] T. Ferraiol, M. Patrão and L. Seco: Jordan decomposition and dynamics on flag manifolds, Discrete Contin. Dyn. Syst. A, 26 No. 3, pp. 923-947, (2010).

[2] Helgason, S. Differential Geometry, Lie Groups and Symmetric Spaces. Academic Press, (1978).

[3] Hoffman, K. and Kunze, R. Linear Algebra. Second Edition. PrenticeHall, (1971). 
[4] Humphreys, J.E. Introduction to Lie Algebras and Representation Theory. Springer, (1972).

[5] Knapp, A. W. Lie Groups Beyond an Introduction, Progress in Mathematics, v. 140, Birkhäuser, (2004).

[6] Mostow, G. D.: Factor Spaces of Solvable Groups. Ann. of Math., 60, No. 1, pp. 1-27, (1954).

[7] Varadarajan, V.S. Lie Groups, Lie Algebras and their Representations. Prentice-Hall Inc., (1974).

[8] Varadarajan, V.S. Harmonic Analysis on Real Reductive Groups. Lecture Notes in Math. 576. Springer-Verlag, 1977.

[9] Warner, G. Harmonic Analysis on Semi-Simple Lie Groups I. Springer-Verlag, (1972).

\section{Mauro Patrão}

Departamento de Matemática

Universidade de Brasília-DF,

Brazil

e-mail : mpatrao@mat.unb.br

\section{Laércio Santos}

Universidade Federal de São Carlos

Campus de Sorocaba

Sorocaba - SP,

Brazil

e-mail : lsantos@ufscar.br

and

\section{Lucas Seco}

Departamento de Matemática

Universidade de Brasília-DF,

Brazil

e-mail : lseco@mat.unb.br 